\title{
CHARACTERISATION OF BLOOD VESSELS USED FOR CORONARY ARTERY BYPASS SURGERY
}

\author{
Marika Garnizone, Edīte VĀrtiña, Māra Pilmane \\ Rīga Stradiņš University, Institute of Anatomy and Anthropology, \\ Department of Morphology, Riga, Latvia
}

\begin{abstract}
Saphenous veins are commonly used as coronary artery bypass grafts. Therefore, it is important to understand the vein wall morphology used for the bypass grafts.

Methods. Ten specimens of saphenous veins were obtained from 10 patients admitted to the Heart Surgery Centre of P. Stradiņš Clinical University Hospital for coronary artery bypass surgery and a histopathological study was conducted. The slides were processed for histological routine and immunohistochemical staining with following markers: endothelin (ET), metallomembranoproteinase 2 (MMP2), tissue inhibitor of metalloproteinase 2 (TIMP2), transforming growth factor beta (TGF $\beta$ ), hepatocyte growth factor (HGF), vascular endothelial growth factor (VEGF), protein gene product 9.5 (PGP9.5), vascular cell adhesion molecule (VCAM), intercellular adhesion molecule (ICAM). For the analysis of the positive structures detected by immunohistochemistry, a semiquantitative evaluation method was used.

Results. Normal histoarchitecture of the vein wall was evaluated by routine staining. Moderate positive endothelin containing endothelial cells and moderate to numerous positive VEGF cells were found on small blood vessels. Moderate positive MMP2 structures and variable - mainly moderate to numerous positive TIMP structures - were found in vein wall. Positive structures were evaluated as equal with both MMP2 and TIMP, with one exception where MMP2 positive structures were evaluated as moderate, but TIMP positive structures as few. All specimens were rich in TGF $\beta$, VCAM and ICAM cells. Abundance of VCAM and ICAM positive endothelial cells was also found. Few HGF positive structures were found in tunica intima and few PGP9.5 positive nerve fibres in tunica adventitia.
\end{abstract}


Conclusions. Rich expression of TGF $\beta$, VCAM, ICAM is characteristic for saphenous vein. A number of VEGF, MMP and TIMP positive cells found in saphenous vein wall indicates a presence of tissue remodelling and ischemia, while low number of HGF positive cells shows its lesser involvement in homeostasis regulation.

Keywords: Vena saphena magna; coronary artery bypass surgery

\section{INTRODUCTION}

The venous system constitutes an adaptable and variable system from person to person and, as such, has been an often-overlooked part of vascular anatomy. Nonetheless, the continued use of veins in operative techniques such as bypasses in addition to highly debilitating manifestations of venous disease stresses the importance of understanding the vein wall and the venous system. In spite of the common basic function of arteries and veins in transporting blood, significant distinctions continue to be found in the organic backgrounds of the two types of vessels.

The venous wall is composed of three layers, the intima, the media, and the adventitia. The internal elastic layer further separates the intima and the media, and the external elastic layer separates the media and adventitia $[1,2]$.

Intima. The intima consists of one layer of simple squamous epithelium endothelium - sitting on an incomplete elastic basement membrane. Under normal healthy conditions, the endothelial cells have essential roles in venous wall integrity and function. These cells produce vasorelaxants, such as prostacyclin (PGI 2) and NO, which are known to prevent platelet activation, adhesion, and aggregation. $\mathrm{NO}$ also negatively affects the expression of chemical mediator secretion and inflammatory cell adhesion molecules including intercellular adhesion molecule-1 (ICAM-1) and vascular cell adhesion molecule-1 (VCAM-1). [1, 3, 4].

In humans, the intima develops only after birth and forms completely by 6 months of age. There is no definite internal elastic lamina and, as in arteries, the thickness of the intima increases with age (phlebosclerosis). In arteries the earliest signs of atherosclerosis and fatty streaks appear in the areas of sheer stress and increased intimal thickness. $[4,5,6]$.

Media. The media is composed of circularly arranged smooth muscle cells and varies greatly according to location. Small veins have only a thin muscular wall, but in larger channels, such as the saphenous vein and the inferior vena cava, there are coarse bundles of irregular muscle, partially organised 
into longitudinal and circular layers $[1,5,7]$. However, in general, it is less well developed than that of the arterial system. This thin media may contribute to the development of varicosities in lower extremity veins. Larger-calibre veins have limited contractile capacity in comparison to the thicker-walled great saphenous vein $[1,5,8]$. The smooth muscle cells of this layer are held in a quiescent state in the normal venous environment by various factors. The tunica media contains extracellular connective elements (elastin, collagen types I and III, proteoglycans, and glycosaminoglycans) and vascular smooth muscle cells. Transforming growth factor- $\beta$ downregulates their mitogenesis and stabilizes the extracellular matrix against smooth muscle cell migration. In addition, heparin and heparin-like molecules neutralize the fibroblast growth factor to downregulate cell proliferation. This process is important because these factors keep the normal vessel wall in a state of low cell turnover with low rates of proliferation and apoptosis. Injury or changes to the environment, as when veins are exposed to arterial flow, can increase the rates of proliferation or apoptosis $[1,9,10]$.

Adventitia. External to a poorly developed external elastic lamina is the adventitia, a loose connection of extracellular matrix with a sparse vasa vasorum. It is generally the thickest layer in large veins. It may sometimes blend with the media. Bundles of longitudinally oriented smooth muscle cells are interspersed with collagen and elastic fibres in this layer. Adventitia may contain bundles of longitudinally oriented smooth muscle cells interspersed with collagen and elastic fibres. The abundant collagen fibres have either a longitudinal or helical orientation. Compared with vasa vasorum of the corresponding arteries, vasa vasorum are much more extensive in venous adventitia and penetrate into deeper regions of the adventitia as well. Lower oxygen tension in venous blood is a possible explanation for this phenomenon $[1,3,10]$.

In general, veins have thinner walls than their corresponding arteries because their cellular and fibrous components are typically more limited than those of the arterial system. The relevance of the difference in the media+adventitia thickness is unknown. It should be noted that the composition of vein walls is also different, with a relative abundance of collagen fibres, particularly in large veins, and a relative paucity of elastic fibres - as might be assumed from the diminished internal and external elastic laminae. Researchers increasingly have recognized the vein graft tunica adventitia as an important repository of progenitor cells which subsequently can migrate and proliferate; and as a source of vascular wall inflammatory cells, cytokines, and chemokines. $[1,11,12,13]$. 


\section{Specific factors of the vein wall}

Endothelin-1 (ET-1) is the most abundant and important endothelin produced by endothelial cells. ET-1 carries out its effects through two types of membrane-G protein-coupled receptors, ET A and ET B. Many important functions are mediated by the activation of these receptors, such as cardiovascular remodelling, vasoconstriction, cell proliferation and differentiation, production of extracellular matrix, and water and sodium secretion control. ET receptors can be found on vascular smooth muscle cells, adventitial fibroblasts, and endothelial cells. $[14,15,16]$.

Matrix metalloproteinases (MMPs), also called matrixins, function in the extracellular environment of cells and degrade both matrix and non-matrix proteins. Matrix metalloproteinases are produced by smooth muscle cells and macrophages to actively modify the matrix. They play central roles in morphogenesis, wound healing, tissue repair and remodelling in response to injury. They are multi-domain proteins and their activities are regulated by tissue inhibitors of metalloproteinases (TIMPs). In addition to an inhibitory role against metalloproteinases, the encoded protein has a unique role among TIMP family members in its ability to directly suppress the proliferation of endothelial cells. As a result, the encoded protein may be critical to the maintenance of tissue homeostasis by suppressing the proliferation of quiescent tissues in response to angiogenic factors, and by inhibiting protease activity in tissues undergoing remodelling of the extracellular matrix $[16,17,18]$.

Transforming growth factor beta (TGF $\beta$ ) encoded protein regulates cell proliferation, differentiation and growth, and can modulate expression and activation of other growth factors. TGF $\beta$ critically regulates the development of neointima formation following vascular injury; however, the underlying signalling mechanisms remain to be fully elucidated. Two distinct type I TGF- $\beta$ receptors, Alk-1 and Alk-5, are both expressed in endothelial cells and pericytes $[17,18,19]$.

Hepatocyte growth factor (HGF) binds to the hepatocyte growth factor receptor to regulate cell growth, cell motility and morphogenesis in numerous cell and tissue types. It also plays a role in angiogenesis, tumorogenesis, and tissue regeneration. Pleiotropic growth factor has potential angiogenic, anti-apoptotic, antifibrotic and anti-inflammatory benefits [17, 20].

Vascular endothelial growth factor (VEGF) induces proliferation and migration of vascular endothelial cells and is essential for both physiological and pathological angiogenesis. Furthermore, stretch-induced modulation of genes involved in myogenic differentiation (VEGF) contributes to the vascular 
remodelling that underlies pathologic complications, such as neointima development and atherosclerosis of the vein grafts. [7, 17, 21].

Protein Gene Product 9.5 (PGP 9.5) is also known as ubiquitin C-terminal hydrolase 1 (UCHL-1). Expression of PGP9.5 is highly specific to neurons and to cells of the diffuse neuroendocrine system. Originally isolated as a neuronspecific protein, it also plays important roles in the nonlysosomal proteolytic pathway $[22,23]$.

Vascular cell adhesion molecule 1 (VCAM-1) encodes a cell surface sialoglycoprotein expressed by cytokine-activated endothelium. This type I membrane protein mediates leukocyte-endothelial cell adhesion and signal transduction and may play a role in the development of atherosclerosis. VCAM-1 expression has been detected on cell types other than endothelium, including macrophages, dendritic cells, astrocytes, bone marrow stromal cells, and respiratory epithelial cell lines $[17,24]$.

Intercellular adhesion molecule 1 (ICAM-1) is a member of the immunoglobulin superfamily of adhesion receptors and is associated with several common diseases, such as heart disease and stroke. It is a cell surface glycoprotein which is typically expressed on endothelial cells and cells of the immune system and is involved in the binding of a cell to another cell or to the extracellular matrix. Levels of endothelial ICAM-1 expression greatly increase after stimulation by cytokines (e.g., IL-1, TNF- $\alpha$, IFN- $\gamma$ ) or bacterial endotoxin. They have roles in cell proliferation, differentiation, motility, trafficking, apoptosis and tissue architecture $[17,24,25]$.

\section{Aim of the study}

The aim of this study was to evaluate morphologic features of healthy saphenous vein used in coronary artery bypass surgery.

\section{MATERIAL AND METHODS}

Vein samples were acquired at the Heart Surgery Centre of Pauls Stradiņš Clinical University Hospital from patients who were admitted to the hospital for coronary artery bypass surgery. The patients' records were retrieved and analysed according to a predetermined protocol.

Ten specimens of saphenous veins were obtained from 10 patients $(7$ males and 3 females) whose age ranged from 55 to 81 years. They had been admitted to the Heart Surgery Centre of Pauls Stradiņš Clinical University Hospital for coronary artery bypass surgery, and a histopathological study was conducted. 
The research was approved by the hospital's Ethics Committee and local Committee of Ethics at Riga Stradiņš University on 22 February 2018. All patients were informed about the procedure and the handling of the received samples.

Data on vascular risk factors were collected from all patients during their visits.

\section{Methods}

Two-to three-centimetre-long tissue fragments were taken from operation material using the less traumatic technique.

The tissue fragments were fixed for 24 hours in Stefanini solution - a mixture consisting of $2 \%$ formaldehyde and $0.2 \%$ picric acid in $0.1-\mathrm{M}$ phosphate buffer ( $\mathrm{pH}$ 7.2) (Stefanini et al., 1967) and were subsequently washed for 12 hours in phosphate buffer ( $\mathrm{pH} 7$ ). Then, tissues were embedded in paraffin and 3-4 $\mu \mathrm{m}$ thick sections were prepared by microtome. The tissue deparaffinization was carried out with xylene, and the tissue was then dehydrated with alcohol $96^{\circ}$. The slides were processed for histological routine straining to obtain an overview picture as well as immunohistochemical staining using the HiDef detection method to identify the following markers in tissue samples: endotelin (mouse, ab-2786, 1:250, Abcam), metalomembranoproteinase 2 (MMP2) (mouse, sc-53630, 1:100, Santa Cruz Biotechnology, Inc), tissue inhibitor of metalloproteinase 2 (TIMP2) (mouse, sc-21735, 1:200, Santa Cruz Biotechnology, Inc), transforming growth factor beta (TGF $\beta$ ) (rabbit, sc-82, 1:100, Santa Cruz Biotechnology, Inc), hepatocyte growth factor (HGF) (goat, f-21, 1:300, RD Systems), vascular endothelial growth factor (VEGF) (rabbit, orv-191500, 1:100, Biorbyt), protein gene product 9.5 (PGP9.5) (rabbit, 439273a, 1:200, Initrogen), vascular cell adhesion molecule (VCAM) (goat, cd-106, 1:200, RD Systems), intercellular adhesion molecule (ICAM) (goat, cd-54, 1:300, RD Systems).

Tissue samples were rinsed with TRIS buffer (Lot 0713513, Diapath S.p.A., Italy) twice for 5 minutes, and placed in boiling EDTA buffer (Lot 0713311, Diapath S.p.A., Italy) in a microwave oven for up to 20 minutes. The container with the samples was then cooled in the buffer for up to 20 minutes, until it reached $65{ }^{\circ} \mathrm{C}$. The specimen was placed in a TRIS wash buffer, and blocking with peroxidase block (Lot 1213603A, Cell Marque, USA) was performed for 10 minutes. The next steps were rinsing twice for 5 minutes as well as rinsing in TRIS wash buffer for 5 minutes. 
For antibodies obtained from goat, the LSAB staining system (Santa Cruz Biotechnology, Inc., USA) was used. First, incubation with the primary antibody for 2 hours was performed, followed by washing in TRIS wash buffer for 5 minutes and then incubation for 30 minutes with biotin (secondary antibody). This process was followed by washing in TRIS buffer for 5 minutes, incubation for 30 minutes with the HRP-streptavidin complex, and then washing in TRIS wash buffer again for 5 minutes. Tissue coating with the liquid $\mathrm{DAB}+$ chromogenic substrate system as well as incubation at room temperature, which together resulted in a positive structure being coloured brown, was carried out for up to 10 minutes. The sample was then washed in distilled water for 5 minutes and counterstained with haematoxylin (Mayer`s haematoxylin, Bio Optica Milano S.p.A., Italy) for 2 minutes.

For antibodies obtained from mouse or rabbit, the EnVision staining system (Lot 1528902C, Cell Marque, USA) was used. Incubation with the primary antibody for 1 hour was followed by washing in TRIS washing buffer twice for 5 minutes and the En Vision+/binding phase for 30 minutes. Washing in TRIS washing buffer twice was then repeated. Tissue coating with liquid DAB+ substrate chromogenic system and incubation at room temperature was performed, resulting in a positive structure having brown coloration; this took between 1-10 minutes, after which the sample was washed in distilled water for 5 minutes and counterstained with haematoxylin (Mayer's haematoxylin, Bio Optica Milano S.p.A., Italy) for 2 minutes.

For the analysis of the positive structures detected by immunohistochemistry, a semiquantitative evaluation method was used (Pilmane et al., 2011). The designations were as follows: 0 - negative reaction; $0 /+-$ occasionally marked structures in the view field; + - a few positive structures in the view field; +/++ a few to moderate number of positive structures in the view field; ++- a moderate number of positive structures found in the view field; $++/+++-$ moderate to numerous positive structures; +++- numerous positive structures in the view field; ++++- abundance of positive structures in view field. The evaluation was performed with a Leica microscope.

\section{RESULTS}

Vein sections revealed normal histoarchitecture of the vein wall composed of 3 tunicae evaluated in routine staining (Fig.1). The first tunica from the luminal side was the intima, which was fully or partially covered with endothelial cells. Tunica intima in all specimens was thin with one exception, where tunica 
intima was thick, wider than tunica media (Fig.2). The media was thick and filled with smooth muscle cells with one exception where the tunica media was thin. The tunica adventitia consisted of bundles of collagen fibres, fibroblasts, vasa vasorum, a few of which were sclerotic in all specimens.

Immunohistochemistry results

Moderate $(++)$ positive endothelin containing endothelial cells (Fig. 3 ) as well as moderate to numerous $(++/+++)$ positive VEGF endothelial cells were found on small blood vessels. Moderate $(++)$ positive MMP2 endothelial cells, smooth muscle cells and fibroblasts (Fig.4) and variable - mainly moderate to numerous $(++/+++)$ positive TIMP endothelial cells, smooth muscle cells and fibroblasts were found. Despite variability, in all cases positive structures were evaluated as equal with both MMP2 and TIMP with one exception where MMP2 positive structures were evaluated as moderate (++), but TIMP positive structures were evaluated as few $(+)$. All specimens were rich in TGF $\beta$, VCAM and ICAM, numerous (+++) TGF $\beta$ structures. Abundance (++++) of VCAM and ICAM positive endothelial cells was also found. HGF expression was not characteristic in veins and only few $(+)$ positive structures were found in tunica intima. Few (+) PGP9,5 positive nerve fibres were found in tunica adventitia.

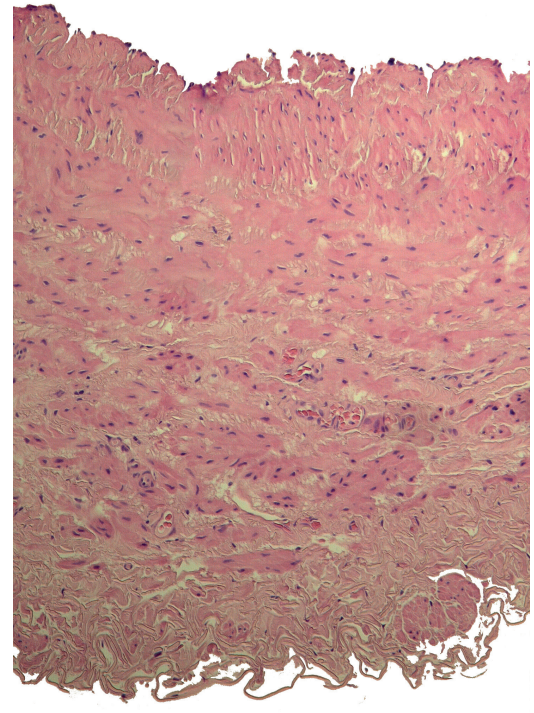

Figure 1. Note classic picture of $v$. saphena magna wall with three intact layers - tunica intima, tunica media and tunica adventitia. Hematoxylin and eosin, $\times 100$

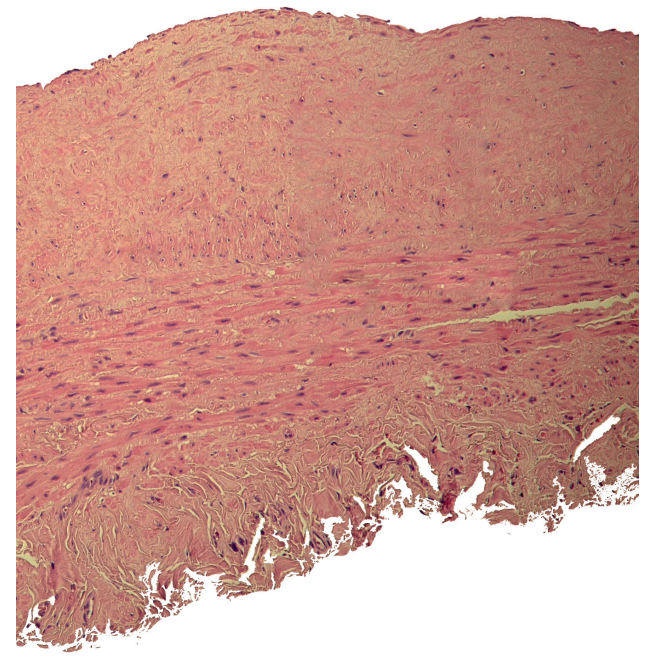

Figure 2. As one exception, see hyperplasia of tunica intima, where it is thick and wider than tunica media. Hematoxylin and eosin, $\times 100$ 


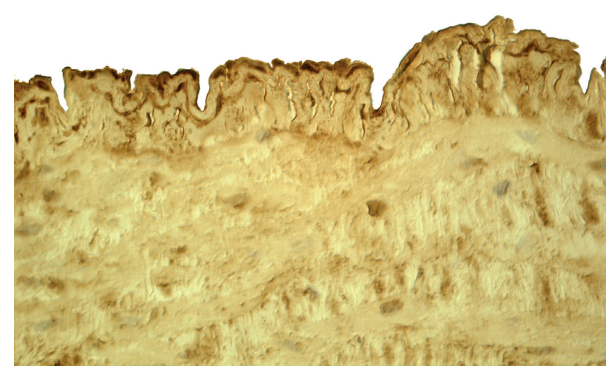

Figure 3. Note in this tunica intima fragment moderate $(++)$ positive endothelin containing endothelial cells. Endothelin IMH, $\times 400$

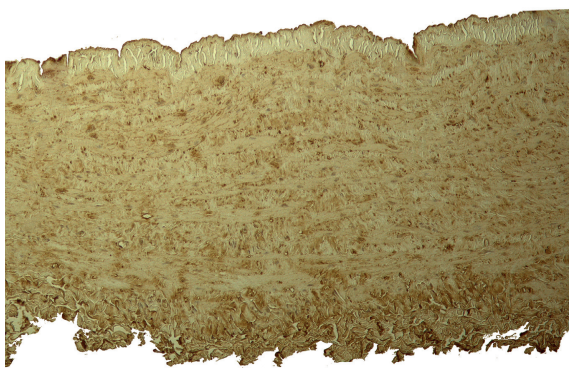

Figure 4. Throughout all layers there can be seen moderate (++) MMP2 positive endothelial cells, smooth muscle cells and fibroblasts. MMP2 IMH, $\times 100$

\section{DISCUSSION}

Moderate endothelin and moderate to numerous VEGF endothelial cells found by us in v.saphena magna wall is characteristic of healthy veins. VEGF is a secreted protein; its binding sites are selectively expressed in endothelial cells in vitro and in vivo; the expression of its mRNA is correlated to blood vessel growth [26]. Endothelin, on the other hand, is considered an important mediator in vessel remodelling, ultimately leading to major changes in cellular and tissue architecture [21].

Equal number of positive MMP2 and TIMP2 endothelial cells, smooth muscle cells and fibroblasts detected in saphenous vein suggests the balance between tissue degeneration enzymes and their inhibitors, while any exception to this model might be a deviation from the ontogenetic norm and plays an important role in vein bypass graft functionality. Mid-term and late saphenous vein graft failure is determined by formation of the neointima [27]. Although, many local and systemic factors have an impact on its development, this process always involves smooth muscle cells located in the tunica media of the saphenous vein segment [28]. Smooth muscle cells release matrix metalloproteinases (MMPs) that degrade extracellular matrix [29]. They also produce tissue inhibitors of metalloproteinases (TIMPs), and a balance between MMPs and TIMPs eventually determines the local homeostasis of the extracellular matrix [30]. In research by Perek et al. (2016) evaluating the expression of proteins, the majority of saphenous vein segments exhibited weak or moderate immunostaining of MMP-2, TIMP-2 and TIMP-3 within the tunica media. The tissue expression of MMP-2 and TIMPs has been associated with the age of the 
patients [31]. The degradation of extracellular matrix by MMPs, which provides a structural framework essential for the functional properties of vessel wall, was found to be a prerequisite for smooth muscle cells migration, a critical step in neointima formation [27]. Moreover, baseline level of MMP-2 in the saphenous vein wall was shown to have a strong linear correlation with the degree of luminal narrowing at 1 year after coronary artery bypass graft [32]. Perek et al. (2016) stated that saphenous vein segments may be more appropriate grafts in the elderly population. Moreover, their study also demonstrated increased tissue activity of TIMP-2 and TIMP- 3 in the tunica media of the saphenous vein segments [31]. However, no correlation between the age of the patient and TIMP-2 activity in the human aortic specimen had been demonstrated [33]. Therefore, it has been suggested that TIMP- 3 is a senescence-related protein [34]. Heavy smoking also noticeably increases native MMP2 and MMP9 gene expression in the saphenous vein before coronary artery bypass graft. Even after long-term cessation of smoking, the dysregulated MMP2 and MMP9 gene expression cannot recover to normal levels. With the elevated native MMP2 and MMP9 gene expression in the saphenous vein induced by heavy smoking, more vein graft disease can be found on long-term follow-up [35].

HGF is not a significant factor in the v.saphena magna wall, because as Gallo et al. (2015) stated, the HGF and its receptor Met pathway has a prominent role in cardiovascular remodelling after tissue injury [36]. However, the abundant deposits of TGF $\beta$, VCAM and ICAM are important for the maintenance of large vein homeostasis. George et al. (2005) suggest that the amount of TGF $\beta$ in human saphenous vein is sufficient, when in the active form, to protect smooth muscle cells against apoptosis. Decreasing the amount of active TGF $\beta$ causes smooth muscle cells to undergo apoptosis, but adding exogenous TGF $\beta$ has no beneficial effect [37], while gene expression of MMP9, MMP12, TIMP1 and TIMP 2 is influenced by the addition of TGF- $\beta 1$. These results may be translated to chronic venous insufficiency framework and suggest involvement of TGF- $\beta 1$ in the vein wall pathology [38].

Saphenous veins used as coronary artery bypass grafts have a specific distribution of the expression of the adhesion molecules ICAM-1 and VCAM-1. These molecules, which belong to the immunoglobulin superfamily, mediate adhesion and transmigration of blood leukocytes through the endothelium. The adhesion of all leukocytes is mediated by the expression of ICAM-1, whereas VCAM-1 is specific for lymphocytes, monocytes, and eosinophils. Adhesion of blood elements to the endothelium is an important step in the development of vein graft disease [39]. The endothelium is immunoregulatory in that inhibiting the function of vascular adhesion molecules blocks leukocyte 
recruitment and, thus, tissue inflammation. Endothelium is stimulated to express adhesion molecules that mediate leukocyte binding. Upon leukocyte binding, these adhesion molecules activate endothelial cell signal transduction that then alters endothelial cell shape for the opening of passageways through which leukocytes can migrate. If the stimulation of this opening is blocked, inflammation is blocked. VCAM-1 on the endothelium functions as both a scaffold for leukocyte migration and a trigger of endothelial signalling [40].

$V$. saphena magna is not richly innervated with neuropeptide-containing nerve fibres. Gulbenkian et al. (1987) discovered that, in the vascular system of guinea pigs, PGP 9.5 immunoreactivity occurred in an extensive plexus of fine perivascular nerve fibres and fascicles running around and along both arteries and veins, mainly at the adventitial-medial border [41]. This shows that only the fascicles of nerve fibres enter the wall of the vein.

\section{CONCLUSIONS}

$V$. saphena magna used for coronary artery bypass surgery possess a moderate number of VEGF and MMP positive cells with slightly more TIMP immunoreactive cells suggesting the connective tissue remodellation and presence of tissue ischemia. Healthy $v$. saphena magna is characterised by rich expression of TGF $\beta$, VCAM and ICAM structures. HGF seems not to be involved in homeostasis regulation of these blood vessels which are slightly innervated by neuropeptide containing nerves.

\section{REFERENCES}

1. Sumpio B., Chin J. (2018). Chapter 3. Vessel Wall Biology. In: Sidawy A.N., Perler B.A. ed. Rutherford's Vascular Surgery and Endovascular Therapy. $9^{\text {th }}$ edition. Philadelphia: Elsevier, 2832.

2. Freischlag J.A., Heller J.A. (2016). Chapter 64. Venous disease. In: Townsend C.M., Beauchamp R.D., Evers, B.M., Mattox, K.L. ed. Sabiston Textbook of Surgery. $20^{\text {th }}$ edition. Philadelphia: Elsevier, 2176.

3. Ovalle W.K., Nahirney P.C. (2013). Chapter 8. Cardiovascular system. In: Ovalle W.K., Nahirney P.C. ed. Netter's Essential Histology. $2^{\text {nd }}$ edition. London: Elsevier, 536.

4. Buratto E., Shi W.Y., Konstantinov I.E. (2016). An intima affair adds to the dominion of the internal thoracic artery in coronary artery bypass grafting. The Journal of Thoracic and Cardiovascular Surgery, 151, 6, 1709-1710. https://doi.org/10.1016/j.jtcvs.2016.02.052 
5. Gallagher P.J., van der Wal A.C. (2013). Chapter 13. Cardiovascular system. In: Cross S. ed. Underwood's Pathology: A Clinical Approach. $6^{\text {th }}$ edition. London: Elsevier, 788.

6. Schwartz S.M., deBlois D., O’Brian E.R. (1995). The intima. Soil for atherosclerosis and restenosis. Circulation Research, 77, 3, 445-465. https://doi.org/10.1161/01.RES.77.3.445

7. Hocking K.M., et al. (2011). Detrimental effects of mechanical stretch on smooth muscle function in saphenous veins. Journal of Vascular Surgery, 53, 2, 454-460. https://doi.org/10.1016/j.jvs.2010.09.010

8. Wei F.C., Al Deek N.F., Lin Tay S.K. (2017). Chapter 24. Principles and techniques of microvascular surgery. In: Gurtner G., Neligan P. ed. Plastic Surgery: Volume 1: Principles. $4^{\text {th }}$ edition, Philadelphia: Elsevier, 888.

9. Airhart N., Curci J.A. (2014). Chapter 9. Arterial Aneurysms. In: Cronenwett J.L., Johnston K.W. ed. Rutherford's Vascular Surgery. $8^{\text {th }}$ edition, London: Elsevier, 2784.

10. Berceli S.A. (2014). Chapter 92. Autogenous Grafts. In: Cronenwett J.L., Johnston K.W. ed. Rutherford's Vascular Surgery. $8^{\text {th }}$ edition. London: Elsevier, 2784.

11. Yu P., et al. (2013). Lack of interleukin-1 signaling results in perturbed early vein graft wall adaptations. Surgery: Official Journal of the Society of University Surgeons. Central Surgical Association, and the American Association of Endocrine Surgeons, 153, 1, 63-69. https://doi.org/10.1016/j.surg.2012.06.005

12. Maiellaro K., Taylor W.R. (2007). The role of the adventitia in vascular inflammation. Cardiovascular Research, 75, 4, 640-648.

https://doi.org/10.1016/j.cardiores.2007.06.023

13. Havelka G.E., Kibbe M.R. (2011). The vascular adventitia: its role in the arterial injury response. Vascular and Endovascular Surgery, 45, 5, 381-390. https://doi.org/10.1177/1538574411407698

14. Trindade M., Oigman W., Fritsch Neves M. (2017). Potential Role of Endothelin in Early Vascular Aging. Current Hypertension Reviews, 13, 1, 33-40. https://doi.org/10.2174/1573402113666170414165735

15. Abraham D., et al. (2008). Endothelin - role in vascular disease. Rheumatology (Oxford, England), 47, 23-24. https://doi.org/10.1093/rheumatology/ken282

16. Velazquez O., Wang B. (2014). Chapter 4. Cells of the Vascular System. In: Cronenwett J.L., Johnston K.W. ed. Rutherford's Vascular Surgery. 8th edition. London: Elsevier, 2784.

17. Life Map Sciences. Gene Cards Suite: The Human Gene Database. 01.02.2018. http://www.genecards.org/.

18. Nagase H., Visse R., Murphy G. (2006). Structure and function of matrix metalloproteinases and TIMPs. Cardiovascular Research, 69, 3, 562-573.

https://doi.org/10.1016/j.cardiores.2005.12.002 
19. Low E.L., Baker A.H., Bradshaw A.C. (2015). Dissecting transforming growth factor-beta signalling pathways in primary human vascular smooth muscle cells. Atherosclerosis, 241, 1, 77. https://doi.org/10.1016/j.atherosclerosis.2015.04.269

20. Lu F., et al. (2013). MSCs transfected with hepatocyte growth factor or vascular endothelial growth factor improve cardiac function in the infarcted porcine heart by increasing angiogenesis and reducing fibrosis. International Journal of Cardiology, 167, 6, 2524-2532. https://doi.org/10.1016/j.ijcard.2012.06.052

21. Shyu K.G., Chang M.L., Wang B.W., Kuan P., Chang H. (2001). Cyclical mechanical stretching increases the expression of vascular endothelial growth factor in rat vascular smooth muscle cells. Journal of Formosan Medical Association (Taiwan yi zhi), 100, 11, 741-747.

22. Biocare medical: PGP9.5, Concentrated Monoclonal Antibody, Control Number: 901-329-011615, 01.02.2018.

https://biocare.net/wp-content/uploads/329.pdf.

23. Kon Y., Endoh D., Iwanaga T. (1999). Expression of protein gene product 9.5, a neuronal ubiquitin C-terminal hydrolase, and its developing change in sertoli cells of mouse testis. Molecular Reproduction and Development, 54, 4, 333-341. https://doi.org/10.1002/(SICI)1098-2795(199912)54:4\%3C333:: AID-MRD3\%3E3.0.CO;2-8

24. Broide D.H., Sriramarao P. (2013). Chapter 6. Cellular Adhesion in Inflammation. In: Franklin Adkinson Jr.N., et al. ed. Middleton's Allergy: Principles and Practice. $8^{\text {th }}$ edition, Philadelphia: Elsevier, 1896.

25. Danik J.S., Paynter N.P., Ridker P.M. (2012). Chapter 21. Genomic Biomarkers in Human Population Studies. In: Ginsburg G.S., Huntington W.F. ed. Genomic and Personalized Medicine. $2^{\text {nd }}$ edition, London: Academic Press, 1350.

26. Ferrara N. (2009). Vascular Endothelial Growth Factor. AHA / ASA Journals: Arteriosclerosis, Thrombosis, and Vascular Biology, 29, 6, 789-791.

https://doi.org/10.1161/ATVBAHA.108.179663

27. Hassantash S.A., et al. (2008). Pathophysiology of aortocoronary saphenous vein bypass graft disease. Asian Cardiovascular \& Thoracic Annals, 16, 4, 331-336. https://doi.org/10.1177/021849230801600418

28. Muto A., et al. (2010). Mechanisms of vein graft adaptation to the arterial circulation - Insights into the neointimal algorithm and management strategies. Circulation Journal: official journal of the Japanese Circulation Society, 74, 8, 1501-1512. https://doi.org/10.1253/circj.CJ-10-0495

29. Johnson J.L., van Eys G.J., Angelini G.D., George S.J. (2001). Injury induces dedifferentiation of smooth muscle cells and increased matrix-degrading metalloproteinase activity in human saphenous vein. Arteriosclerosis, Thrombosis, Vascular Biology, 21, 7, 1146-1151. https://doi.org/10.1161/hq0701.092106

30. Raffetto J.D., Khalil R.A. (2008). Matrix metalloproteinases and their inhibitors in vascular remodeling and vascular disease. Biochemical Pharmacology, 75, 2, 346-359. https://doi.org/10.1016/j.bcp.2007.07.004 
31. Perek B., et al. (2016). Potentially positive ageing-related variations of medial smooth muscle cells in the saphenous veins used as aortocoronary bypass grafts. Folia Histochemica Cytobiologica, 54, 2, 91-98.

https://doi.org/10.5603/FHC.a2016.0011

32. Kon Z.N., et al. (2007). The role of preexisting pathology in the development of neointimal hyperplasia in coronary artery bypass grafts. The Journal of Surgical Research, 142, 2, 351-356. https://doi.org/10.1016/j.jss.2007.03.062

33. McNulty M., Spiers P., McGovern E., Feely J. (2005). Aging is associated with increased matrix metalloproteinase- 2 activity in the human aorta. American Journal of Hypertension, 18, 4 Pt 1, 504-509. https://doi.org/10.1016/j.amjhyper.2004.11.011

34. Kamei M., Hollyfield J.G. (1999). TIMP-3 in Bruch's membrane: changes during aging and in age-related macular degeneration. Investigative Ophthalmology \& Visual Science, 40, 10, 2367-2375.

35. Yongxin S., et al. (2013). Heavy smoking before coronary surgical procedures affects the native matrix metalloproteinase- 2 and matrix metalloproteinase- 9 gene expression in saphenous vein conduits. The Annals of Thoracic Surgery, 95, 1, 55-61. https://doi.org/10.1016/j.athoracsur.2012.08.073

36. Gallo S., Sala V., Gatti S., Crepaldi T. (2015). Cellular and molecular mechanisms of HGF/Met in the cardiovascular system. Clinical Science (London, England: 1979), 129, 12, 1173-1193. https://doi.org/10.1042/CS20150502

37. George S.J., et al. (2005). Transforming growth factor-beta is activated by plasmin and inhibits smooth muscle cell death in human saphenous vein. Journal of Vascular Research, 42, 3, 247-254. https://doi.org/10.1159/000085657

38. Serralheiro P., et al. (2017). Effect of TGF-betal on MMP/TIMP and TGF-beta1 receptors in great saphenous veins and its significance on chronic venous insufficiency. Phlebology, 32, 5, 334-341. https://doi.org/10.1177/0268355516655067

39. Chester A.H., Morrison K.J., Yacoub M.H. (1998). Expression of vascular adhesion molecules in saphenous vein coronary bypass grafts. The Annals of Thoracic Surgery, 65, 6, 1685-1689. https://doi.org/10.1016/S0003-4975(98)00274-4

40. Cook-Mills J.M., Marchese M.E., Abdala-Valencia H. (2011). Vascular cell adhesion molecule- 1 expression and signaling during disease: regulation by reactive oxygen species and antioxidants. Antioxidants \& Redox Signaling, 15, 6, 1607-1638. https://doi.org/10.1089/ars.2010.3522

41. Gulbenkian S., Wharton J., Polak J.M. (1987). The visualisation of cardiovascular innervation in the guinea pig using an antiserum to protein gene product 9.5 (PGP 9.5). Journal of the Autonomic Nervous System, 18, 3, 235-247. https://doi.org/10.1016/0165-1838(87)90122-6 
Address for correspondence

Prof. Māra Pilmane

Rīga Stradiņš University

Institute of Anatomy and Anthropology

Department of Morphology

E-mail: mara.pilmane@rsu.lv

Marika Garnizone

Rīga Stradiṇš University

Institute of Anatomy and Anthropology

Department of Morphology

E-mail: marika.garnizone@gmail.com 